\title{
Differences in Anticoagulant Therapy Prescription in Patients with Paroxysmal Versus Persistent Atrial Fibrillation: Insights from the NCDR ${ }^{\circledR}$ PINNACLE Registry
}

\author{
Jonathan C. Hsu, MD, MAS ${ }^{\star}$, Paul S. Chan, MD, MSc ${ }^{\dagger}$, Fengming Tang, MS $^{\dagger}$, Thomas M. \\ Maddox, MD, MSc ${ }^{\ddagger}$, and Gregory M. Marcus, MD, MAS $\S$ \\ "Cardiac Electrophysiology Section, Division of Cardiology, Department of Medicine, University of \\ California, San Diego, San Diego, California \\ †Mid America Heart Institute, Kansas City, Missouri \\ ‡VA Eastern Colorado Health Care System/University of Colorado School of Medicine, Denver, \\ Colorado \\ §ection of Cardiac Electrophysiology, Division of Cardiology, Department of Medicine, University \\ of California, San Francisco, San Francisco, California
}

\section{Abstract}

Objectives-We sought to examine whether there are differences in rates of appropriate oral anticoagulant treatment among patients with paroxysmal versus persistent atrial fibrillation (AF) in real-world cardiology practices.

\begin{abstract}
Background-Patients with paroxysmal AF experience a similar risk of thromboembolism compared to patients with persistent AF. Therefore, consensus guidelines recommend anticoagulant therapy in those at risk for thromboembolism irrespective of AF classification.
\end{abstract}

Methods-We identified outpatients with AF and intermediate to high thromboembolic risk $\left(\mathrm{CHADS}_{2}\right.$ score 22$)$ enrolled in the American College of Cardiology PINNACLE Registry between July, 2008 and June, 2012. Using hierarchical modified Poisson regression models adjusted for patient characteristics, we examined whether anticoagulant treatment rates differed between patients with paroxysmal versus persistent AF.

Results-Of 71,972 patients, 56,513 (78.5\%) had paroxysmal and 15,459 (21.5\%) had persistent AF. In both unadjusted and multivariable adjusted analyses, patients with paroxysmal AF were less frequently prescribed oral anticoagulant therapy than those with persistent AF (50.4\% vs. $64.3 \%$; adjusted risk ratio [RR] 0.74, $95 \%$ confidence interval [CI] 0.72-0.76). Instead, patients

Address for Correspondence: Jonathan C. Hsu, MD, MAS, 9444 Medical Center Dr., MC7411, La Jolla, CA, 92037, Fax: (858) 657-5314, Phone: (858) 657-5310, Jonathan.Hsu@ucsd.edu.

Disclosures:

Dr. Paul S. Chan is supported by a Career Development Grant Award (K23HL102224) from the National Heart Lung and Blood Institute.

Dr. Gregory M. Marcus receives research support from Medtronic, Baylis Medical, Gilead, and SentreHeart Inc.

Dr. Maddox is supported by a Health Services Research and Development career development award from the U.S. Department of Veterans Affairs. 
with paroxysmal AF, compared with those with persistent $\mathrm{AF}$, were more frequently prescribed only antiplatelet therapy (35.1\% vs. $25.0 \%$; adjusted RR $1.79,95 \%$ CI $1.70-1.88$ ) or neither antiplatelet nor anticoagulant therapy ( $14.5 \%$ vs. $10.8 \%$; adjusted RR $1.34,95 \%$ CI $1.26-1.44$; $P<0.0001$ for differences across all 3 comparisons).

Conclusions-In a large, real-world cardiac outpatient population, patients with paroxysmal with a moderate to high risk of stroke were less likely to be prescribed appropriate oral anticoagulant therapy and more likely to be prescribed less effective or no therapy for thromboembolism prevention.

\section{Keywords}

Atrial fibrillation; Anticoagulants; Paroxysmal; Persistent; Stroke; Thromboembolism

Atrial fibrillation (AF) is the most common cardiac arrhythmia with an estimated 1 in 4 lifetime risk in those older than 40 years of age, and a large projected increase in prevalence by the year $2050(1,2)$. In patients at risk for thromboembolism, full oral anticoagulation with warfarin (a vitamin $\mathrm{K}$ antagonist) or the newer novel anticoagulants reduces morbidity and mortality, regardless of AF duration or permanence (7-10). Although AF can be either paroxysmal ( $<7$ days duration), or persistent, studies have found that a patient's risk of stroke is independent of AF type $(3,4)$. As a result, current treatment guidelines recommend anticoagulant therapy in patients at moderate to high risk for thromboembolism irrespective of AF classification (11). However, it remains unclear it remains unclear if patients with paroxysmal AF are less aggressively treated with effective oral anticoagulants compared with patients with persistent AF.

Accordingly, we examined anticoagulation treatment patterns between patients with paroxysmal and persistent AF in a contemporary cohort of outpatients using data from the National Cardiovascular Data Registry (NCDR)'s Practice Innovation and Clinical Excellence (PINNACLE) Registry ${ }^{\circledR}$. Use of this prospective and contemporary national registry of cardiovascular care in the United States, provides a unique opportunity to examine patterns of oral anticoagulant treatment in routine practice among outpatients in whom anticoagulant treatment is not affected by acute illness.

\section{Methods}

\section{Data Source}

The NCDR PINNACLE registry was created in 2008 by the American College of Cardiology as the first national, prospective, office-based cardiac quality improvement registry in the United States $(12,13)$. Participating academic and private practices collect longitudinal, point of care data that includes patient demographics, symptoms, comorbidities, vital signs, medications, laboratory values, and recent hospitalizations with either paper forms, or modification of a practice's electronic medical record using a standardized collection tool to comprehensively obtain and transmit uniform data. Quality checks and analyses of the data are performed at St. Luke's Mid America Heart Institute (Kansas City, Missouri), the primary analytical center for the PINNACLE registry. 


\section{Study Population}

Of 1,711,326 patients enrolled into the PINNACLE registry between July 1, 2008 and June $30,2012,359,315$ had a diagnosis of AF. We included only those 108,890 patients in whom AF duration (paroxysmal versus persistent) was specified. We further restricted the cohort to patients known to be at moderate to high risk for thromboembolism $\left(\mathrm{CHADS}_{2}\right.$ score $\left.\geq 2\right)$ $[\mathrm{n}=36,918$ excluded] and patients from cardiology practices with $\geq 10$ eligible patients [XXX patients from 36 practices excluded]. Moreover, we excluded patients with a documented medical, patient, or system contraindication to any oral anticoagulant $(n=4,717)$. Therefore, our final study cohort was comprised of 71,972 patients from XX practices with known AF duration at moderate to high risk for thromboembolism.

To minimize over-representation by patients with multiple visits, only data from the index visit of each patient were used. Since the $\mathrm{CHA}_{2} \mathrm{DS}_{2}$-VASc score has also been shown to be more effective tool to risk-stratify patients at highest risk (and therefore to derive the greatest benefit) from antiacogulant therapy (6), we also compared anticoagulant treatment patterns for a $\mathrm{CHA}_{2} \mathrm{DS}_{2}$-VASc score $\geq 2$. This exploratory analysis led to the inclusion of an additional 27,058 AF patients, or an analytical cohort of 99,030 patients with a $\mathrm{CHA}_{2} \mathrm{DS}_{2-}$ VASc score 2 .

\section{Study Variables and Outcomes}

The main independent variable was type of AF, defined in PINNACLE as either paroxysmal or persistent (with no distinction between persistent vs. permanent AF). Our main study outcome was treatment with any oral anticoagulant, which would include warfarin or one of the recently approved novel anticoagulants (dabigatran or rivaroxaban, as apixaban had not yet been approved the U.S. Food and Drug Administration during the study timeframe). Among patients not treated with anticoagulant therapy, we also examined whether these patients were treated with an antiplatelet agent or were not receiving either anticoagulant or antiplatelet therapy. Treatment with an antiplatelet agent was defined as prescription of aspirin, clopidogrel, ticlopidine, prasugrel and/or dipyridamole.

\section{Statistical Analysis}

Unadjusted differences in baseline demographic and clinical characteristics were compared between patients with paroxysmal and persistent AF using the $\chi^{2}$ test for categorical variables and t-tests for continuous variables.

To examine whether anticoagulant treatment patterns differed between patients with paroxysmal and persistent AF, we constructed hierarchical modified Poisson regression models, adjusted for patient demographic and clinical characteristics. These models included site as a random effect to account for patient clustering within sites. Covariates considered to be potential confounders were entered as fixed effects in the model and included age, sex, U.S. geographical region, health insurance, body mass index (BMI), $\mathrm{CHADS}_{2}$ score, congestive heart failure, hypertension, diabetes, prior stroke/transient ischemic attack, systemic embolism, coronary artery disease, prior myocardial infarction, recent coronary artery bypass grafting or percutaneous coronary intervention, stable angina, peripheral arterial disease, dyslipidemia, and tobacco use. 
Race was not included in the model due to a high rate of missing data for race (_._\%). Besides race, the highest missing rate for other variables included BMI (27.7\%), tobacco use (25.3\%), and insurance payer (16.2\%), and the average number of missing data field per patient was X.X. Missing data were assumed to be missing at random and were imputed with 10 imputation data sets (14), in which all patient variables were used to inform the imputation model (15). We performed an exploratory analysis that also imputed and included race in each model, which did not significantly alter each model's point estimate of the study outcomes.

Since the rate of oral anticoagulant prescription, antiplatelet therapy prescription, and failure to treat with any antithrombotic therapy all exceeded 10\%, we used modified Poisson regression models at all steps to estimate relative risks (RRs) directly (instead of odds ratios obtained from logistic regression, which may overestimate effect differences) (16,17). In $a$ priori secondary analyses, we also examined whether antithrombotic treatment in paroxysmal versus persistent AF patients differed within specific subgroups, including patients of different age ( $\geq 75$ years versus $<75$ years), sex, and CHADS $_{2}$ score, as well as patients with and without congestive heart failure and coronary artery disease. An interaction $p$ value $<0.05$ in subgroup analyses was considered statistically significant. Furthermore, we repeated all above analyses in the expanded cohort of 99,030 patients with a $\mathrm{CHA}_{2} \mathrm{DS}_{2}$-VASc score $\geq 2$.

All statistical tests were evaluated at a 2-sided significance level of 0.05. Analyses were performed using the SAS statistical package version 9.2 (SAS Institute, Cary, NC), R version 2.7.0 (Foundation for Statistical Computing, Vienna, Austria), and IVEWare (Institute for Social Research, University of Michigan, Ann Arbor).

\section{Results}

Of 71,972 AF patients with moderate to high risk of stroke, 56,513 (78.5\%) had paroxysmal AF and 15,459 (21.5\%) had persistent AF. A comparison of demographic and clinical characteristics between patients with paroxysmal and persistent AF is shown in Table 1. Paroxysmal AF patients were younger and more frequently of white race and female sex. Compared with those with persistent $\mathrm{AF}$, patients with paroxysmal $\mathrm{AF}$ had a higher $\mathrm{CHA}_{2} \mathrm{DS}_{2}$ score $(2.9 \pm 1.0$ vs. $2.7 \pm 1.0 ; \mathrm{P}<0.001)$ and a higher $\mathrm{CHA}_{2} \mathrm{DS}_{2}-\mathrm{VASC}$ score (X.X \pm X.X vs. X.X \pm X.X; $\mathrm{P}<0.001$ ). They were also more likely to reside in the Northeast, be active smokers, and have private health insurance, diabetes, prior stroke or transient ischemic attack, coronary artery disease, prior myocardial infarction, recent CABG or PCI, stable angina and peripheral arterial disease, and tobacco use. In contrast, patients with paroxysmal AF were less likely to have coexisting heart failure.

A total of 39,196 (54.5\%) AF patients were prescribed an oral anticoagulant. Among these, warfarin was the most commonly used therapy ( $\mathrm{n}=34,937$ [89.1\%]), followed by dabigatran $(n=3,423[8.7 \%])$ and rivaroxaban $(n=836$ [2.1\%]). In unadjusted analysis, compared to those with persistent $\mathrm{AF}$, patients with paroxysmal $\mathrm{AF}$ were less frequently prescribed oral anticoagulant therapy (50.4\% versus 64.3\%). Instead, patients with paroxysmal AF were more frequently prescribed only antiplatelet therapy (35.1\% versus $25.0 \%)$ or were untreated 
for their thromboembolic risk (14.5\% versus 10.8\%) (Table 2). After multivariable adjustment for differences in patient characteristics in the 2 groups, these differences persisted. Patients with paroxysmal AF were less likely to be appropriately treated with oral anticoagulant therapy (adjusted risk ratio $[\mathrm{RR}] 0.74,95 \%$ confidence interval $[\mathrm{CI}] 0.72$ 0.76). Instead, they were more likely to be treated with either antiplatelet therapy only (adjusted RR $1.79,95 \%$ CI 1.70-1.88, $\mathrm{p}<0.0001$ ) or no antithrombotic therapy at all (adjusted RR 1.34, 95\% CI 1.26-1.44, $P<0.0001$ across the 3 treatment categories) (Figure 1). In prespecified subgroup analysis of the primary cohort, the likelihood of treatment with antithrombotic therapy in paroxysmal versus persistent AF patients was consistent across most subgroups studied (Figure 2A-C). However, patients with congestive heart failure were more likely to be treated with oral anticoagulation and less likely to either be treated with antiplatelet therapy or untreated, regardless of AF subtype ( $\mathrm{p}<0.05$ for all interaction terms). Additionally, younger patients $<75$ years were particularly more likely to receive antiplatelet therapy only ( $\mathrm{p}$ value interaction $=0.003$, Figure $2 B$ ).

In the expanded cohort of 99,030 PINNACLE registry patients with a $\mathrm{CHA}_{2} \mathrm{DS}_{2}$-VASc score $\geq 2$, there was a similar pattern of less aggressive anticoagulant treatment among eligible patients with paroxysmal AF: oral anticoagulant therapy (48.3\% versus $60.2 \%$ ), antiplatelet therapy only (36.0\% versus $26.4 \%)$, and no antithrombotic therapy (15.6\% versus $13.3 \% ; P<0.001)$. As before after multivariable adjustment, patients with paroxysmal AF were less likely to be treated with any oral anticoagulant therapy (adjusted RR $0.72,95 \%$ CI 0.70-0.74; $\mathrm{p}<0.0001$ ), and more likely to be treated with antiplatelet therapy only (adjusted RR 1.89, 95\% CI 1.81-1.97, p<0.0001) or no antithrombotic therapy at all (adjusted RR 1.27, 95\% CI 1.20-1.34, p<0.0001).

\section{Discussion}

In a large, nationally representative sample of 71,972 outpatients with $\mathrm{AF}$ at intermediate to high risk of stroke $\left(\mathrm{CHADS}_{2}\right.$ score 2 ) treated by cardiologists, patients with paroxysmal compared to persistent AF were $>25 \%$ less likely to be prescribed oral anticoagulant therapy, $>75 \%$ more likely to be prescribed antiplatelet only therapy, and $>30 \%$ more likely to be untreated with any antithrombotic therapy after adjustment for comorbidities. These findings were consistent in an expanded population of 99,030 outpatients with $\mathrm{AF}$ and at least a moderate risk of stroke using a different metric $\left(\mathrm{CHA}_{2} \mathrm{DS}_{2}-\mathrm{VASc}\right.$ score 2$)$. Our findings have important implications, particularly as stroke risk is considered equivalent between paroxysmal and persistent AF patients, with clear guideline-based recommendations for oral anticoagulation based on risk factors regardless of AF type or duration.

Previous studies including the SPAF (Stroke Prevention in Atrial Fibrillation III) trial showed similar rates of ischemic stroke during aspirin treatment in patients with paroxysmal (3.2\%) and permanent (3.3\%) AF (3). Additionally, the ACTIVE W (Atrial Fibrillation Clopidogrel Trial With Irbesartan for Prevention of Vascular Events) substudy showed a similar annualized risk of stroke in both paroxysmal (2.0\%) and persistent (2.2\%) AF patients that were no different in those assigned to oral anticoagulation (4). Recent work from the ASSERT (Asymptomatic Atrial Fibrillation and Stroke Evaluation in Pacemaker 
Patients and the Atrial Fibrillation Reduction Atrial Pacing Trial) investigators showed that even short, subclinical episodes of AF are associated with ischemic stroke or systemic embolism (18).

We found that only $54.5 \%$ of AF patients at moderate to high risk of stroke were prescribed an oral anticoagulant, a proportion similar to the $59-70 \%$ of AF patients that did not receive appropriate oral anticoagulation reported by other groups (19-21). Previous studies investigating predictors of appropriate oral anticoagulation in AF patients have primarily included AF subtype classification only as a covariate. In one study of 945 inpatients with $\mathrm{AF}$, older age and a perceived bleeding risk of the patient increased the risk of failure to receive appropriate anticoagulation, whereas persistent or permanent $\mathrm{AF}$ and prior thromboembolism predicted treatment with oral anticoagulation (22). Among 5,333 hospitalized and ambulatory AF patients in Europe, valvular heart disease, persistent or permanent $\mathrm{AF}$, and diabetes predicted a higher likelihood of oral anticoagulation prescription, whereas an alternative reason for hospital admission, major bleeding, and lack of an oral anticoagulation monitoring clinic predicted a lower likelihood of oral anticoagulation prescription (23). A single center study of 572 patients found that paroxysmal and persistent AF patients were almost 3 times as likely to be prescribed oral anticoagulation, but the reference group was "transitory" AF patients with a single episode of AF without recurrence (24). In general, these previous studies have not specifically focused on AF sub-type as a primary predictor, nor have they focused on the outpatient care of AF patients by cardiologists guided by specialty-defined treatment guidelines.

We found that outpatient cardiologists in modern practice were more than $25 \%$ less likely to prescribe oral anticoagulant therapy, and more than $75 \%$ more likely to be prescribe antiplatelet therapy only, to paroxysmal AF patients at risk for stroke. Although paroxysmal and persistent AF patients differed by several characteristics and comorbidities, we accounted for potential confounding by extensive multivariable adjustment and found that these associations persisted. Since paroxysmal AF patients may more often present to an outpatient clinic in normal sinus rhythm, it is plausible that cardiologists taking care of these patients are less apt to prescribe oral anticoagulation based only on a documented history of intermittent AF that is not present at the time of an encounter. Similarly, since AF is more likely to be captured by an electrocardiogram at the time of an outpatient encounter in persistent/permanent AF patients, this finding may sway prescribing physicians to better adhere to guideline-based recommendations due to a "seeing is believing" phenomena. Or, perhaps due to an intuitive belief that less AF means a lower risk of stroke, treating physicians may find it easier and less risky to prescribe antiplatelet therapy only to patients who are predominantly in sinus rhythm despite a clear benefit of oral anticoagulation over antiplatelet therapy in clinical trials backed by consensus guidelines $(11,25)$.

We performed additional analyses to assure the robustness of our findings. We studied an expanded cohort of AF patients with a $\mathrm{CHA}_{2} \mathrm{DS}_{2}$-VASc score $\geq 2$, as this more risk scheme (6) may improve discrimination of AF patients at risk for stroke and thromboembolism (26). Our findings consistently showed that paroxysmal compared to persistent AF patients were less likely to be prescribed appropriate oral anticoagulant therapy, demonstrating disproportionate guideline non-adherence in paroxysmal AF patients across the spectrum of 
thromboembolic risk. Since a contraindication to oral anticoagulation in this large registry could not be confirmed for each patient, all patients were included in the original analysis. However, when we performed a sub-analysis excluding patients with a provider documented medical, patient, or system contraindication to any oral anticoagulant prescription, we consistently observed similar guideline adherence failures in paroxysmal AF patients. These findings suggest provider bias in the prescription of pharmacotherapy to reduce stroke risk in paroxysmal AF patients.

Both congestive heart failure and younger age appeared to modify the likelihood of guideline-based treatment of paroxysmal compared to persistent AF patients. In most subgroups (e.g. patients of each sex, with and without coronary artery disease, and across different $\mathrm{CHADS}_{2}$ scores) paroxysmal AF patients consistently experienced a lower likelihood of receiving guideline-based therapy compared to persistent AF patients. However, patients with heart failure were more often treated according to guidelines. This may demonstrate that cardiologists recognize heart failure as a particularly powerful predictor of stroke and thromboembolism in AF, regardless of subtype. This might also reflect the influence of subspecialists, such as electrophysiologists who may more often see these patients for implantable cardioverter-defibrillators or cardiac resynchronization devices and may be most familiar with guidelines related to AF. Additionally, younger patients $<75$ years were particularly more likely to receive antiplatelet therapy only, suggesting that a combination of young age and paroxysmal AF may influence prescribing physician perception of thromboembolic risk despite other risk factors that would mandate full oral anticoagulation.

\section{Study limitations}

Our study has several limitations. First, the PINNACLE program enrolled patients from motivated cardiology practices dedicated to quality improvement. Therefore, antithrombotic therapy prescription patterns among paroxysmal and persistent AF patients in other U.S. practices may differ from those reported in this study, potentially reducing the generalizability of our results. However, in general, the PINNACLE program has captured data from over _ practices in __ states, and should be considered one of the largest data repositories available with the breadth to answer the study questions posed. Second, the subtype (paroxysmal versus persistent) of AF was not available for a large amount of patients with AF in the PINNACLE Registry, who therefore could not be included in our study. Differential missing rates of paroxysmal versus persistent AF patients who were included in our study as a result could have led to potential confounding in our study findings. Third, the PINNACLE registry does not collect data regarding oral anticoagulant dose, or monitoring results of warfarin use, which limits our ability to provide insight as to whether paroxysmal versus persistent AF patients were treated differently in terms of the quality or appropriateness of anticoagulant therapy once prescribed. Fourth, specific data are unavailable regarding previous bleeding complications or exact reasons for contraindications to anticoagulant therapy, and therefore we cannot determine the validity of a reported contraindication. However, any bleeding complications or contraindication to antithrombotic therapy would likely be non-differential in respect to paroxysmal versus persistent AF and therefore unlikely to bias our results. Moreover, we performed a sub-analysis excluding 
patients with a reported contraindication to oral anticoagulation, which did not alter our study findings.

\title{
Conclusions
}

In a large, real-world national registry of AF outpatients who were at intermediate to high risk of stroke, patients with paroxysmal compared to persistent AF were less often prescribed guideline-based treatment with oral anticoagulation and more often prescribed antiplatelet only therapy or not treated with any antithrombotic therapy at all. These findings draw attention to important gaps in the appropriate treatment of paroxysmal AF patients and highlight opportunities to improve appropriate prescription of oral anticoagulation in all $\mathrm{AF}$ patients at-risk for thromboembolism.

\section{Acknowledgments}

\author{
None \\ Funding Sources:
}

This research was supported by the American College of Cardiology Foundation's National Cardiovascular Data Registry (NCDR). The views expressed in this manuscript represent those of the authors, and do not necessarily represent the official views of the NCDR or its associated professional societies identified at www.ncdr.com. PINNACLE Registry ${ }^{\circledR}$ is an initiative of the American College of Cardiology Foundation. Bristol-Myers Squibb and Pfizer Inc. are Founding Sponsors of the PINNACLE Registry.

\section{Abbreviations List}
AF
Atrial fibrillation
CI
Confidence interval
NCDR
National Cardiovascular Data Registry
PINNACLE Practice Innovation and Clinical Excellence
RR
Risk ratio

\section{References}

1. Lloyd-Jones DM, Wang TJ, Leip EP, et al. Lifetime risk for development of atrial fibrillation: the Framingham Heart Study. Circulation. 2004; 110:1042-6. [PubMed: 15313941]

2. Go AS, Hylek EM, Phillips KA, et al. Prevalence of diagnosed atrial fibrillation in adults: national implications for rhythm management and stroke prevention: the AnTicoagulation and Risk Factors in Atrial Fibrillation (ATRIA) Study. Jama. 2001; 285:2370-5. [PubMed: 11343485]

3. Hart RG, Pearce LA, Rothbart RM, McAnulty JH, Asinger RW, Halperin JL. Stroke with intermittent atrial fibrillation: incidence and predictors during aspirin therapy. Stroke Prevention in Atrial Fibrillation Investigators. J Am Coll Cardiol. 2000; 35:183-7. [PubMed: 10636278]

4. Hohnloser SH, Pajitnev D, Pogue J, et al. Incidence of stroke in paroxysmal versus sustained atrial fibrillation in patients taking oral anticoagulation or combined antiplatelet therapy: an ACTIVE W Substudy. J Am Coll Cardiol. 2007; 50:2156-61. [PubMed: 18036454]

5. Gage BF, Waterman AD, Shannon W, Boechler M, Rich MW, Radford MJ. Validation of clinical classification schemes for predicting stroke: results from the National Registry of Atrial Fibrillation. Jama. 2001; 285:2864-70. [PubMed: 11401607] 
6. Lip GY, Nieuwlaat R, Pisters R, Lane DA, Crijns HJ. Refining clinical risk stratification for predicting stroke and thromboembolism in atrial fibrillation using a novel risk factor-based approach: the euro heart survey on atrial fibrillation. Chest. 2010; 137:263-72. [PubMed: 19762550]

7. van Walraven C, Hart RG, Singer DE, et al. Oral anticoagulants vs aspirin in nonvalvular atrial fibrillation: an individual patient meta-analysis. JAMA. 2002; 288:2441-8. [PubMed: 12435257]

8. Hart RG, Pearce LA, Aguilar MI. Meta-analysis: antithrombotic therapy to prevent stroke in patients who have nonvalvular atrial fibrillation. Ann Intern Med. 2007; 146:857-67. [PubMed: 17577005]

9. Connolly SJ, Ezekowitz MD, Yusuf S, et al. Dabigatran versus warfarin in patients with atrial fibrillation. N Engl J Med. 2009; 361:1139-51. [PubMed: 19717844]

10. Patel MR, Mahaffey KW, Garg J, et al. Rivaroxaban versus warfarin in nonvalvular atrial fibrillation. N Engl J Med. 2011; 365:883-91. [PubMed: 21830957]

11. Fuster V, Ryden LE, Cannom DS, et al. ACC/AHA/ESC 2006 Guidelines for the Management of Patients with Atrial Fibrillation: a report of the American College of Cardiology/American Heart Association Task Force on Practice Guidelines and the European Society of Cardiology Committee for Practice Guidelines (Writing Committee to Revise the 2001 Guidelines for the Management of Patients With Atrial Fibrillation): developed in collaboration with the European Heart Rhythm Association and the Heart Rhythm Society. Circulation. 2006; 114:e257-354. [PubMed: 16908781]

12. Chan PS, Oetgen WJ, Buchanan D, et al. Cardiac performance measure compliance in outpatients: the American College of Cardiology and National Cardiovascular Data Registry's PINNACLE (Practice Innovation And Clinical Excellence) program. J Am Coll Cardiol. 2010; 56:8-14. [PubMed: 20620710]

13. Chan PS, Oetgen WJ, Spertus JA. The Improving Continuous Cardiac Care (IC(3)) program and outpatient quality improvement. Am J Med. 2010; 123:217-9. [PubMed: 20193826]

14. Harrell, FE. Regression modeling strategies : with applications to linear models, logistic regression, and survival analysis. New York: Springer; 2001.

15. Raghunathan, TE.; Solenberger, PW.; Van Hoewyk, J. IVEware: imputation and variance estimation software. Ann Arbor, MI: Survey Methodology Program, Survey Research Center, Institute for Social Research, University of Michigan; 2002.

16. Greenland S. Model-based estimation of relative risks and other epidemiologic measures in studies of common outcomes and in case-control studies. Am J Epidemiol. 2004; 160:301-5. [PubMed: $15286014]$

17. Zou G. A modified poisson regression approach to prospective studies with binary data. Am J Epidemiol. 2004; 159:702-6. [PubMed: 15033648]

18. Healey JS, Connolly SJ, Gold MR, et al. Subclinical atrial fibrillation and the risk of stroke. N Engl J Med. 2012; 366:120-9. [PubMed: 22236222]

19. Go AS, Hylek EM, Borowsky LH, Phillips KA, Selby JV, Singer DE. Warfarin use among ambulatory patients with nonvalvular atrial fibrillation: the anticoagulation and risk factors in atrial fibrillation (ATRIA) study. Ann Intern Med. 1999; 131:927-34. [PubMed: 10610643]

20. Reynolds MR, Shah J, Essebag V, et al. Patterns and predictors of warfarin use in patients with new-onset atrial fibrillation from the FRACTAL Registry. Am J Cardiol. 2006; 97:538-43. [PubMed: 16461052]

21. Gallagher AM, Rietbrock S, Plumb J, van Staa TP. Initiation and persistence of warfarin or aspirin in patients with chronic atrial fibrillation in general practice: do the appropriate patients receive stroke prophylaxis? J Thromb Haemost. 2008; 6:1500-6. [PubMed: 18573187]

22. Waldo AL, Becker RC, Tapson VF, Colgan KJ. Hospitalized patients with atrial fibrillation and a high risk of stroke are not being provided with adequate anticoagulation. J Am Coll Cardiol. 2005; 46:1729-36. [PubMed: 16256877]

23. Nieuwlaat R, Capucci A, Lip GY, et al. Antithrombotic treatment in real-life atrial fibrillation patients: a report from the Euro Heart Survey on Atrial Fibrillation. Eur Heart J. 2006; 27:301826. [PubMed: 16731536]

24. Glazer NL, Dublin S, Smith NL, et al. Newly detected atrial fibrillation and compliance with antithrombotic guidelines. Arch Intern Med. 2007; 167:246-52. [PubMed: 17296879] 
25. Connolly S, Pogue J, Hart R, et al. Clopidogrel plus aspirin versus oral anticoagulation for atrial fibrillation in the Atrial fibrillation Clopidogrel Trial with Irbesartan for prevention of Vascular Events (ACTIVE W): a randomised controlled trial. Lancet. 2006; 367:1903-12. [PubMed: 16765759]

26. Olesen JB, Torp-Pedersen C, Hansen ML, Lip GY. The value of the CHA2DS2-VASc score for refining stroke risk stratification in patients with atrial fibrillation with a CHADS2 score 0-1: a nationwide cohort study. Thromb Haemost. 2012; 107:1172-9. [PubMed: 22473219] 


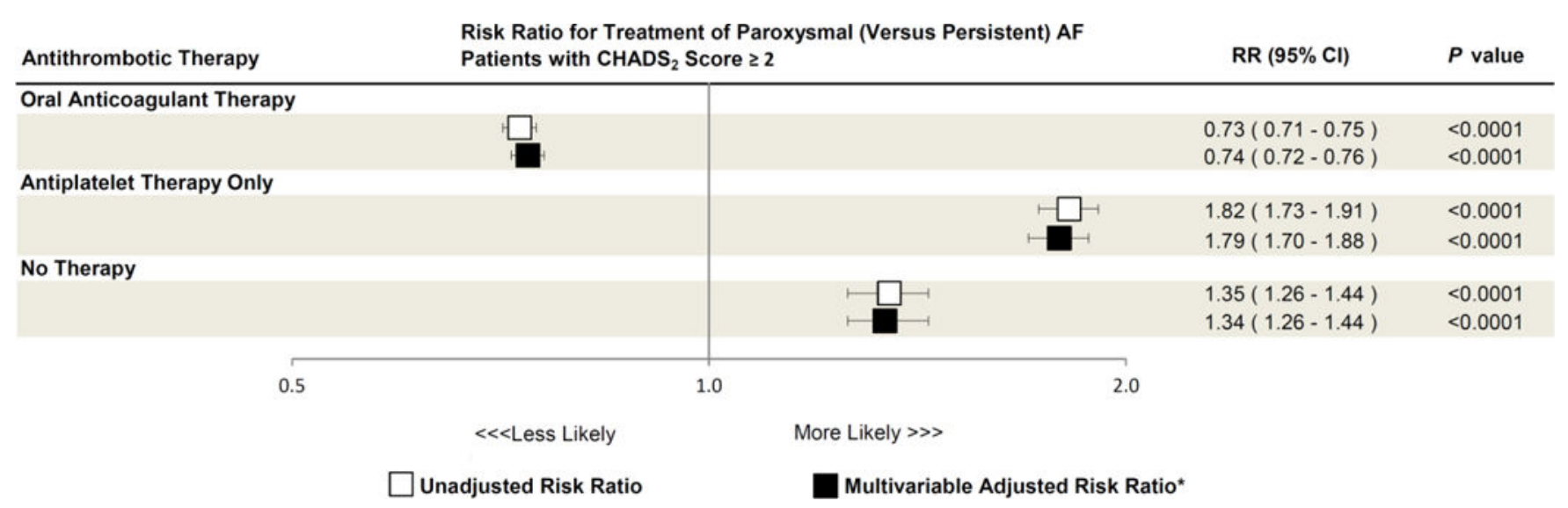

Figure 1. Association between Paroxysmal versus Persistent Atrial Fibrillation Classification and Likelihood of Antithrombotic Prescription in Patients with a Moderate to High Risk of Stroke

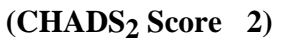

Unadjusted (white boxes) and multivariable adjusted (black boxes) risk ratios of oral anticoagulant therapy, antiplatelet only therapy, and no therapy prescription in paroxysmal versus persistent atrial fibrillation (AF) patients are shown. Error bars denote $95 \%$ confidence intervals.

Oral anticoagulant therapy was defined as prescription of either warfarin, dabigatran, or rivaroxaban. Antiplatelet therapy was defined as prescription of either individual or combination of aspirin, clopidogrel, ticlopidine, prasugrel, and/or dipyridamole.

$\mathrm{CI}=$ confidence interval; $\mathrm{RR}=$ risk ratio.

*Adjusted for age, sex, region, insurance, body mass index (BMI), $\mathrm{CHADS}_{2}$ score, congestive heart failure, hypertension, diabetes, prior stroke/transient ischemic attack, systemic embolism, coronary artery disease, prior myocardial infarction, recent coronary artery bypass grafting or percutaneous coronary intervention, stable angina, peripheral arterial disease, dyslipidemia, and tobacco use. 


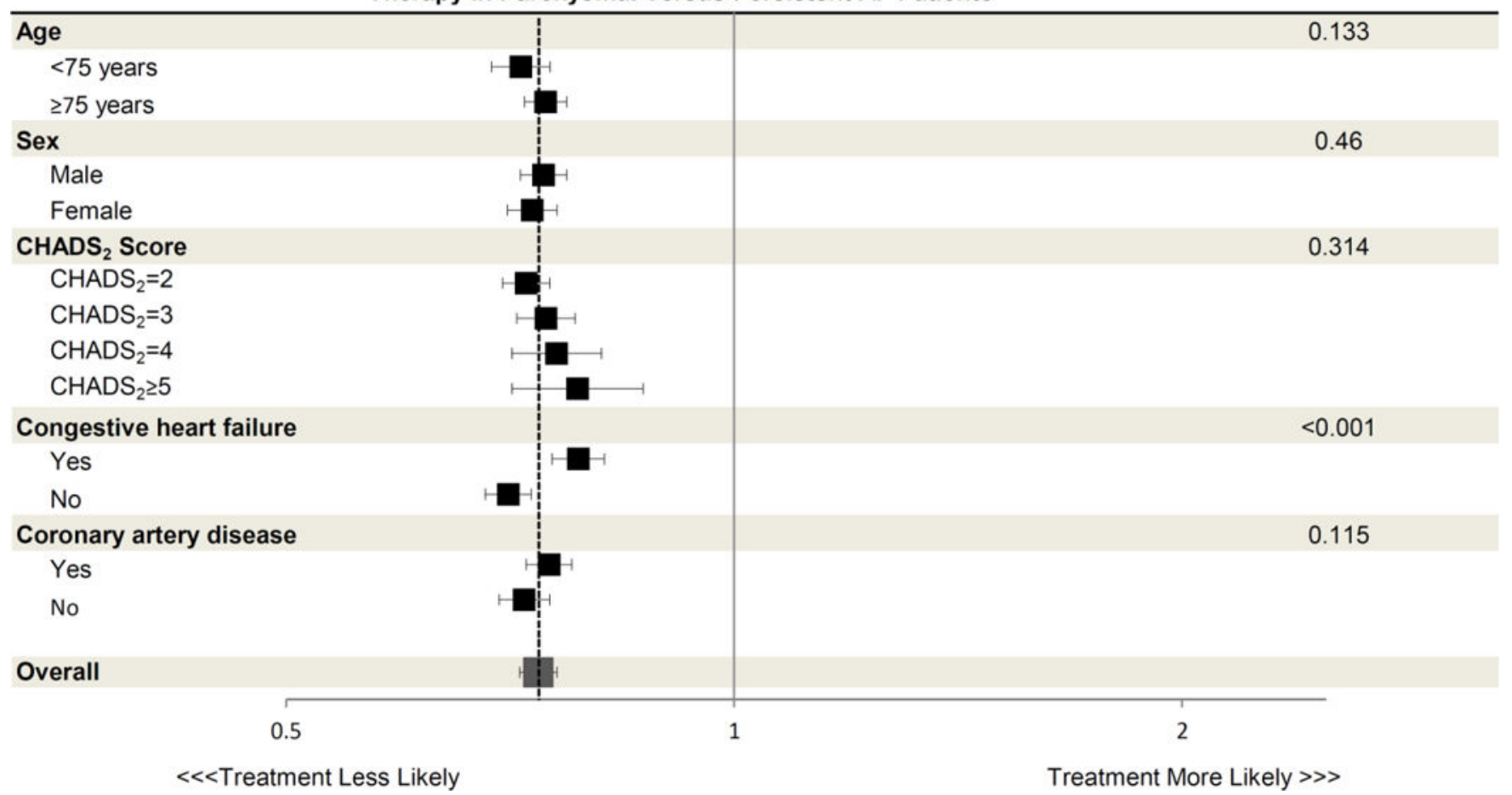


B

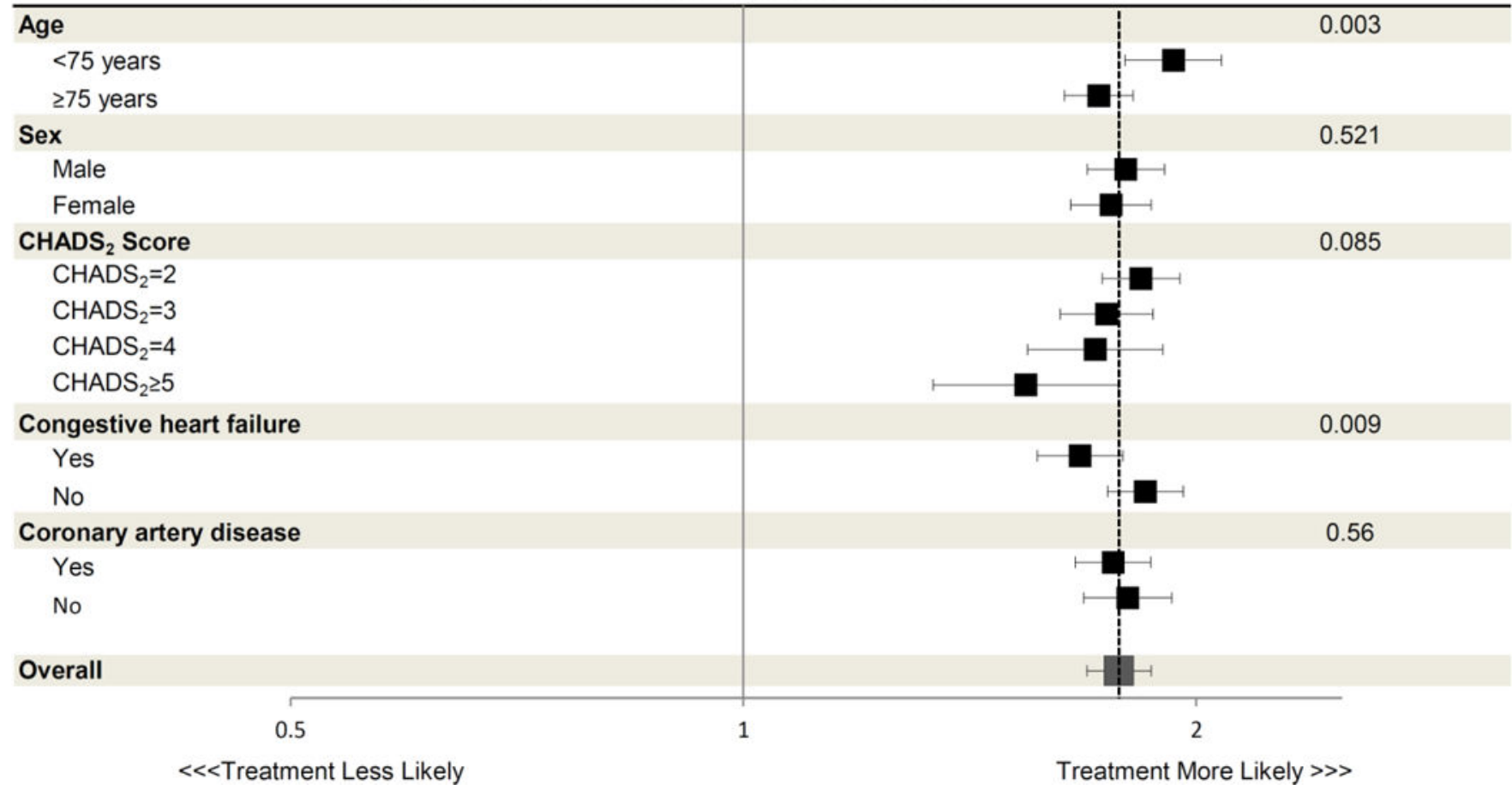




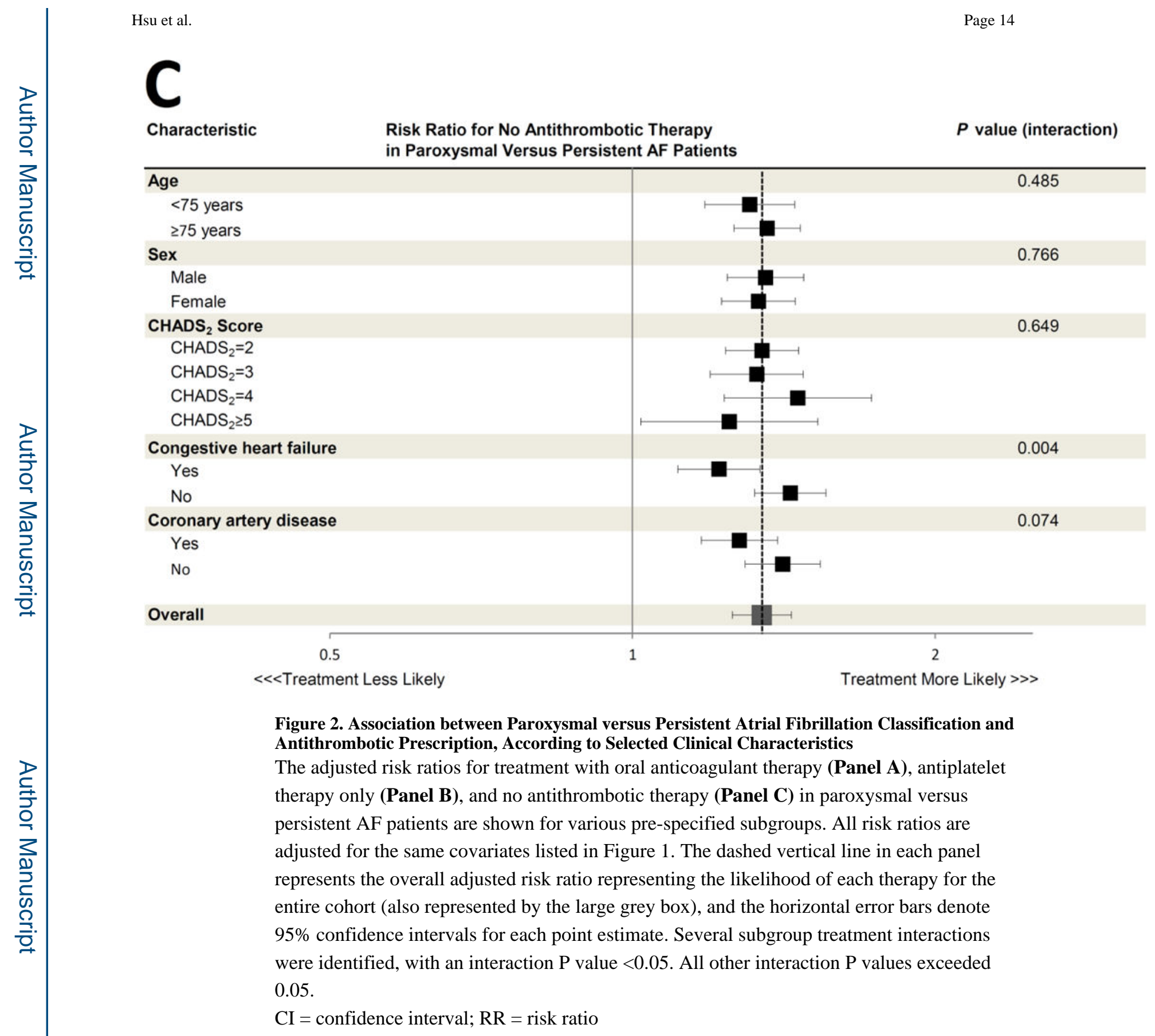


Table 1

Baseline Characteristics of Paroxysmal versus Persistent Atrial Fibrillation Patients with a Moderate to High Risk of Stroke $\left(\mathrm{CHADS}_{2}\right.$ Score 22$)$

\begin{tabular}{|c|c|c|c|}
\hline Characteristic & $\begin{array}{l}\text { Paroxysmal Atrial Fibrillation } \\
(\mathbf{N}=\mathbf{5 6 , 5 1 3})\end{array}$ & $\begin{array}{l}\text { Persistent Atrial Fibrillation } \\
(\mathrm{N}=\mathbf{1 5 , 4 5 9 )}\end{array}$ & p Value \\
\hline \multicolumn{4}{|c|}{ Patient demographic characteristics } \\
\hline Age, years & $75.0 \pm 11.4$ & $76.9 \pm 10.8$ & $<0.001$ \\
\hline Male sex & $51.4 \%$ & $55.0 \%$ & $<0.001$ \\
\hline Race & & & $<0.001$ \\
\hline White & $93.6 \%$ & $91.9 \%$ & \\
\hline Black & $5.0 \%$ & $7.1 \%$ & \\
\hline Other & $1.5 \%$ & $1.1 \%$ & \\
\hline Hispanic ethnicity & $5.9 \%$ & $3.5 \%$ & $<0.001$ \\
\hline Insurance & & & $<0.001$ \\
\hline Private & $54.6 \%$ & $46.4 \%$ & \\
\hline Medicare & $38.0 \%$ & $49.8 \%$ & \\
\hline Medicaid & $1.0 \%$ & $0.8 \%$ & \\
\hline Other & $0.6 \%$ & $0.3 \%$ & \\
\hline Uninsured & $5.8 \%$ & $2.6 \%$ & \\
\hline Region & & & $<0.001$ \\
\hline Northeast & $14.0 \%$ & $5.5 \%$ & \\
\hline Midwest & $32.2 \%$ & $51.8 \%$ & \\
\hline South & $32.3 \%$ & $27.7 \%$ & \\
\hline West & $21.4 \%$ & $15.0 \%$ & \\
\hline Body mass index & $29.2 \pm 6.7$ & $29.2 \pm 6.7$ & 0.980 \\
\hline \multicolumn{4}{|l|}{ Comorbidities } \\
\hline $\mathrm{CHADS}_{2}$ Score & $2.9 \pm 1.0$ & $2.7 \pm 0.9$ & $<0.001$ \\
\hline Congestive heart failure & $38.8 \%$ & $50.9 \%$ & $<0.001$ \\
\hline Hypertension & $93.2 \%$ & $93.1 \%$ & 0.693 \\
\hline Diabetes & $34.1 \%$ & $30.4 \%$ & $<0.001$ \\
\hline Prior stroke/TIA & $31.9 \%$ & $15.8 \%$ & $<0.001$ \\
\hline Systemic embolism & $1.7 \%$ & $1.4 \%$ & 0.014 \\
\hline Coronary artery disease & $65.5 \%$ & $59.2 \%$ & $<0.001$ \\
\hline Prior myocardial infarction & $31.0 \%$ & $23.8 \%$ & $<0.001$ \\
\hline Recent CABG or PCI & $23.9 \%$ & $13.4 \%$ & $<0.001$ \\
\hline Stable angina & $8.4 \%$ & $7.7 \%$ & 0.013 \\
\hline Peripheral arterial disease & $15.8 \%$ & $12.2 \%$ & $<0.001$ \\
\hline Dyslipidemia & $65.1 \%$ & $66.0 \%$ & 0.051 \\
\hline Tobacco use & & & $<0.0001$ \\
\hline Never & $32.8 \%$ & $47.4 \%$ & \\
\hline Current & $15.0 \%$ & $10.7 \%$ & \\
\hline Quit within 12 months & $2.6 \%$ & $1.4 \%$ & \\
\hline
\end{tabular}




\begin{tabular}{cccc} 
Characteristic & $\begin{array}{c}\text { Paroxysmal Atrial Fibrillation } \\
(\mathbf{N = 5 6 , 5 1 3 )}\end{array}$ & $\begin{array}{c}\text { Persistent Atrial Fibrillation } \\
(\mathbf{N = 1 5 , 4 5 9 )}\end{array}$ & p Value \\
\hline Quit more than 12 months ago & $49.6 \%$ & $40.5 \%$ & \\
\hline
\end{tabular}

Categorical data are reported as percentages. Continuous data are reported as mean $\pm \mathrm{SD}$.

$\mathrm{CABG}=$ coronary artery bypass grafting; $\mathrm{PCI}=$ percutaneous coronary intervention; $\mathrm{TIA}=$ transient ischemic attack. 
Table 2

Prevalence of Antithrombotic Prescription in Paroxysmal versus Persistent Atrial Fibrillation Patients with a Moderate to High Risk of Stroke $\left(\mathrm{CHADS}_{2}\right.$ Score $\left.\geq 2\right)$

\begin{tabular}{lcc} 
Therapy & $\begin{array}{c}\text { Paroxysmal Atrial Fibrillation } \\
(\mathbf{N = 5 6 , 5 1 3})\end{array}$ & $\begin{array}{c}\text { Persistent Atrial Fibrillation } \\
(\mathbf{N = 1 5 , 4 5 9 )}\end{array}$ \\
\hline Antithrombotic therapy & & $<0.001$ \\
Any oral anticoagulant therapy only & $26.3 \%$ & $36.1 \%$ \\
Any oral anticoagulant therapy and any antiplatelet therapy & $24.1 \%$ & $28.2 \%$ \\
Any antiplatelet therapy only & $35.1 \%$ & $25.0 \%$ \\
No therapy & $14.5 \%$ & $10.8 \%$ \\
\hline
\end{tabular}

Categorical data are reported as percentages.

Oral anticoagulant therapy was defined as prescription of either warfarin, dabigatran, or rivaroxaban. Antiplatelet therapy was defined as prescription of either individual or combination of aspirin, clopidogrel, ticlopidine, prasugrel, and/or dipyridamole. 